\title{
Hydrogen Isotope Exchange in Proton-Conducting Oxides During Proton and Deuteron Irradiation"
}

\author{
V. B. Vykhodets ${ }^{a}$, T. E. Kurennykh ${ }^{a}$, V. P. Gorelov $b$, V. B. Balakireva ${ }^{b}$, \\ E. V. Vykhodets ${ }^{c}$, and S. I. Obukhov ${ }^{a}$ \\ ${ }^{a}$ Institute of Metal Physics, Ural Division, Russian Academy of Sciences, Yekaterinburg, 620990 Russia \\ ${ }^{b}$ Institute of High-Temperature Electrochemistry, Ural Division, Russian Academy of Sciences, Yekaterinburg, 620219 Russia \\ ${ }^{c}$ Ural Federal University, Yekaterinburg, 6200002 Russia \\ Received March 20, 2013
}

\begin{abstract}
It has been found that during accelerator beam deuteron irradiation of a proton-conducting oxide containing protium H/D isotope exchange between beam ions and dissolved ions takes place. This isotope exchange was also observed during high-energy proton irradiation of the oxide containing dissolved deuterium atoms. These results provide evidence to a new type of hydrogen isotope exchange. Any appreciable effects of conjugate diffusion of hydrogen and oxygen ions and of the interface processes on the isotope exchange rate were eliminated. In this type of exchange the rate of replacement of $\mathrm{H}+$ by $\mathrm{D}^{+}$and of $\mathrm{D}^{+}$by $\mathrm{H}^{+}$was determined only by the properties of the crystal. The discovered effect was used in our study to obtain experimental data characterizing the dynamic and equilibrium behavior of hydrogen isotopes in the oxide $\mathrm{BaZr}_{0.9} \mathrm{Y}_{0.1} \mathrm{O}_{3-\delta}$.
\end{abstract}

DOI: $10.1134 / \mathrm{S} 0021364013110106$

\section{INTRODUCTION}

Oxide materials with high proton conduction are promising functional materials for fuel cells, separators, and hydrogen sensors, as well as for other applications related to nuclear and hydrogen power engineering. Therefore, the data on hydrogen solubility and diffusion, hydrogen isotope exchange, and the corresponding $\mathrm{H} / \mathrm{D} / \mathrm{T}$ isotope effects for these systems are of considerable practical and theoretical interest. Their examination is an effective tool for receiving the information about the states of atoms in crystals and about the mechanism of their diffusion. However, in the case of solid solutions of hydrogen isotopes in oxides, such experimental data are scarce, and their influence on the modern ideas about the state of hydrogen ions in oxides is only speculative in nature. For example, no experimental data on hydrogen diffusion in oxides obtained by direct methods are available in the literature. Likewise, we are aware of only one work where the H/D isotope effect on solubility was measured and only in one oxide at one temperature [1]. An anomalous and very strong isotope effect during $\mathrm{H} / \mathrm{D}$ and $\mathrm{D} / \mathrm{H}$ isotope exchange in proton-conducting oxide ceramics was reported in other studies [2-4].

The lack of data concerning hydrogen isotope exchange in oxides is due mainly to the fact that classic isotope exchange experiments involve treating an oxide containing hydrogen isotopes, for example protium, in a medium from which another isotope (deuterium) comes in contact with the sample. The medium

\footnotetext{
"The article was translated by the authors.
}

is usually humid air containing water vapor. In this case, adsorption, chemisorption, dissociation, and formation of $\mathrm{H}_{2} \mathrm{O}, \mathrm{D}_{2} \mathrm{O}$ or $\mathrm{HD}$ molecules can take place at the oxide surface. Each of these processes is characterized by its own isotope effect. In addition, conjugate diffusion of oxygen and hydrogen ions will generally take place in the sample itself. As a result, experimentally observed isotope effects in the kinetics of replacement of $\mathrm{H}^{+}$by $\mathrm{D}^{+}$or of $\mathrm{D}^{+}$by $\mathrm{H}+$ appear to be cumulative and difficult to quantify. Probably, the anomalous results during $H / D$ and $D / H$ isotope exchange observed in [2-4] are due to these challenges.

Hypothesis: a new type of the hydrogen isotope exchange. In this work, we propose a method to overcoming these challenges by studying H/D isotope exchange under conditions that eliminate the conjugate diffusion of oxygen and hydrogen atoms and the significant influence of interface processes on the isotope exchange rate. In other words, we will control the experimental conditions such that the rate of replacement of $\mathrm{H}^{+}$by $\mathrm{D}^{+}$and vice-versa will be determined mainly by processes occurring inside the crystal.

The method proposed to examine H/D isotope exchange is based on producing oxide samples that contain dissolved protium and deuterium ions. These samples are placed under proton or deuteron irradiation. Here we do not exclude the scenario in which $\mathrm{H}^{+}$ ions in the crystal will be replaced by $\mathrm{D}^{+}$ions or $\mathrm{D}^{+}$ ions by $\mathrm{H}^{+}$ions, while the total number of protium and deuterium ions in the analysis area will be practically constant. The physical reason for the hydrogen isotope 
exchange scenario during deuteron or proton irradiation can be the fulfillment of the electroneutrality condition: during irradiation, the cationic subsystem of the sample receives an excess positive charge, and a possible mechanism of charge compensation is the rapid transition of excess hydrogen ions from the sample to vacuum. The specific purpose of this work was justifying or disproving the supposition about the new hydrogen isotope exchange scenario in oxides during high-energy deuteron and proton irradiation, analyzing H/D isotope effects in the kinetics of this new isotope exchange and obtaining information on the dynamic and equilibrium properties of hydrogen isotopes in oxides.

We measured concentration of deuterium $C_{\mathrm{D}}$ in oxides and the data on dependence of $C_{\mathrm{D}}$ upon deuteron and proton irradiation dose was used as a criterion for the new hydrogen isotope exchange scenario. For instance, the generally expected result of deuteron irradiation is the growth of deuterium concentration $C_{\mathrm{D}}$ in the sample with an increase in the irradiation dose $N_{\mathrm{d}}$. The shape of the dependence of $C_{\mathrm{D}}$ upon $N_{\mathrm{d}}$ may signify the mechanism of the process. For hydrogen isotope exchange, in particular, we write the following equation

$$
\begin{gathered}
d C_{\mathrm{D}}=k_{1} C_{\mathrm{H}} d N_{\mathrm{d}}, \\
C_{\mathrm{H}}+C_{\mathrm{D}}=\text { const }=C_{0},
\end{gathered}
$$

where $d C_{\mathrm{D}}$ is the variation of deuterium concentration in the analysis area at the irradiation dose $d N_{\mathrm{d}}$; the constant $k_{1}$ characterizes the probability of the isotope exchange event during replacement of $\mathrm{H}^{+}$by $\mathrm{D}^{+} ; C_{0}$ is the total concentration of hydrogen isotopes in the initial (unirradiated) sample. From Eqs. (1), (2) at $N_{\mathrm{d}} \longrightarrow 0$, it is easy to derive

$$
\partial C_{\mathrm{D}} / \partial N_{\mathrm{d}}=k_{1}\left(C_{0}-C_{\text {Din }}\right),
$$

where $C_{\text {Din }}$ is the concentration of deuterium ions in the initial sample. The proton irradiation process can be considered in a similar way: using the hypothesis for replacement of $\mathrm{D}^{+}$ions by $\mathrm{H}^{+}$in the crystal at $N_{\mathrm{p}} \longrightarrow 0$, it is easy to deduce

$$
\partial C_{\mathrm{D}} / \partial N_{\mathrm{p}}=-k_{2} C_{\text {Din }},
$$

where $k_{2}$ is the probability of replacement of $\mathrm{D}^{+}$by $\mathrm{H}^{+}$ ions. Equations (3) and (4) can be experimentally verified with the nuclear reaction analysis method (NRA). To verify the hypothesis of the new hydrogen isotope exchange scenario it is necessary to obtain samples with approximately equal total concentration $C_{0}$ of protium and deuterium ions, but with different concentration $C_{\text {Din }}$ in the initial samples.

\section{SAMPLES AND METHODS}

Experiments were performed using $\mathrm{BaZr}_{0.9} \mathrm{Y}_{0.1} \mathrm{O}_{3-\delta}$ oxide micropowders. This oxide is a proton conductor. The powder was prepared according to the standard high-temperature ceramic technology from ultrapure substances $\mathrm{BaCO}_{3}, \mathrm{ZrO}_{2}$, and $\mathrm{Y}_{2} \mathrm{O}_{3}$. X-ray phase analysis showed that the samples were single phases with a cubic perovskite structure. The sintered samples were ground dry in a sapphire mortar and pestle. The specific surface area of the powder was measured to be $2.4 \mathrm{~m}^{2} / \mathrm{g}$.

The resulting micropowders were treated in moist air atmospheres containing saturated water vapors. Under these conditions structural oxygen vacancies in the oxide were replaced by oxygen ions that entered the samples with water molecules. According to study [5], in the special annealing conditions, the concentration of structural oxygen vacancies in $\mathrm{BaZr}_{0.9} \mathrm{Y}_{0.1} \mathrm{O}_{3-\delta}$ should reach a value close to zero, i.e., the maximum possible saturation of the oxide with water molecules should be reached. Thus, the expected composition of the samples upon different treatment conditions in moist air atmospheres was $\mathrm{BaZr}_{0.9} \mathrm{Y}_{0.1} \mathrm{O}_{3} \mathrm{H}_{x} \mathrm{D}_{0.1-x}$. Water with a natural mixture of hydrogen isotopes, deuterium-isotope-rich heavy water, and their mixtures in different ratios were used in the experiments to obtain the initial samples with different concentrations of protium and deuterium. The hydrogen subsystem of heavy water contained $99.8 \%$ of deuterium. A temperature of water was $40^{\circ} \mathrm{C}$. The powder was saturated with water vapor first for $20 \mathrm{~h}$ at $400^{\circ} \mathrm{C}$ and then for $40 \mathrm{~h}$ at $300^{\circ} \mathrm{C}$. Further increase in the annealing time did not lead to increases in deuterium concentration. Below, the total concentration of hydrogen isotopes corresponding to this formula is assumed to be 100 at $\%$ for convenience. In this manner, we produced powders with nine different deuterium concentrations, including the samples containing mainly only protium $(x \approx 0.1)$ or only deuterium ions $(x \approx 0)$. Control measurements showed that the samples were stable, i.e., the concentration of deuterium did not decrease during one-week storage of the samples at room temperature in air or in vacuum.

The concentration of deuterium $C_{\mathrm{D}}$ was measured using the NRA method. Specifically, a 2 MV Van de Graaff accelerator and the ${ }^{2} \mathrm{H}(\mathrm{d}, \mathrm{p})^{3} \mathrm{H}$ reaction with incident beam particles with an energy of $650 \mathrm{keV}$ were used. To obtain the dependence of $C_{\mathrm{D}}$ on $N_{\mathrm{p}}$, proton irradiation was carried out at the proton energy $540 \mathrm{keV}$ since the projected range depth of protons at this energy is almost the same as that of deuterons at $650 \mathrm{keV}[6,7]$. In order to measure the concentration of deuterium during proton irradiation, we used the following procedure: proton irradiation was interrupted for a short period of time, during which the concentration $C_{\mathrm{D}}$ was measured using the reaction ${ }^{2} \mathrm{H}(\mathrm{d}, \mathrm{p}){ }^{3} \mathrm{H}$. The diameter of the incident beam was $2 \mathrm{~mm}$. The accelerator beam current in all the experiments was about $300 \mathrm{nA}$ and it was maintained constant with an accuracy of $20 \%$. The examined samples were placed perpendicular to the incident beam axis and the nuclear reaction product acceptance of the silicon surface barrier detector was $160^{\circ}$. To perform the 


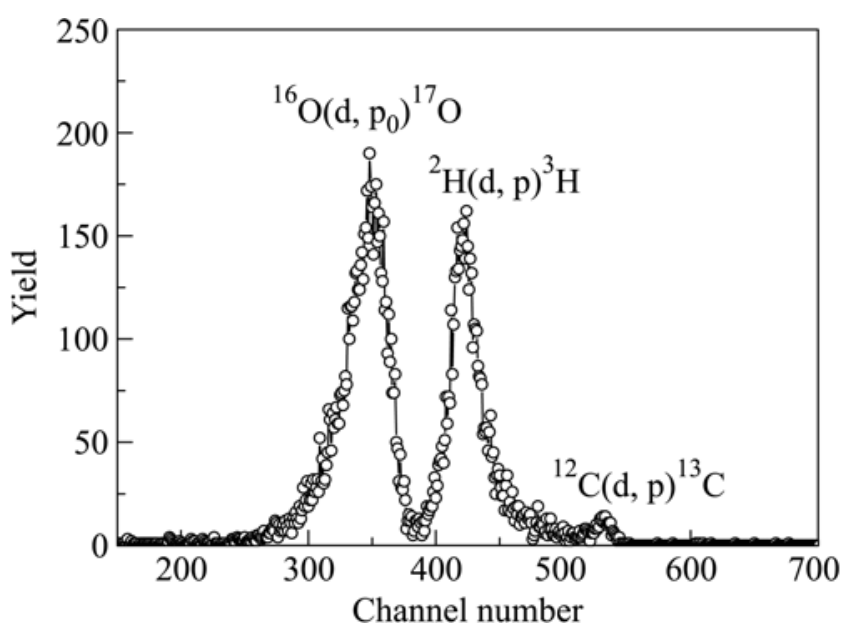

Fig. 1. Typical spectrum of the nuclear reaction products.

measurements, the powder particles were pressed into a soft indium plate. The surface layer consisted only of the oxide particles [8]. We measured the average deuterium concentration near the sample surface to a depth of about $3 \mu \mathrm{m}$. Oxide layer on the surface of the indium plate was thicker than $3 \mu \mathrm{m}$ [8]. A typical spectrum of the nuclear reaction products is shown in Fig. 1.

Two methods for measuring the irradiation doses $N_{\mathrm{p}}$ and $N_{\mathrm{d}}$ were employed. In the case of protons, we used a secondary monitor based on the measurement of the number of protons backscattered by a thin gold film. The film was placed on a propeller blade, which periodically interrupted the beam directed to the sample. The statistical error in the determination of $N_{\mathrm{p}}$ with this method was $\sim 1 \%$. During deuteron irradiation we also used a secondary monitor, which is based on the measurement of the number of protons formed as a result of the reaction ${ }^{16} \mathrm{O}\left(\mathrm{d}, \mathrm{p}_{0}\right){ }^{17} \mathrm{O}$ in the samples. The statistical error in the determination of $N_{\mathrm{d}}$ with this method was less than $0.15 \%$. Both secondary monitors were calibrated using a Faraday cup.

The primary data were mathematically treated by comparing the spectra from the samples examined with those of a reference sample of the deuteride micropowder $\mathrm{ZrCr}_{2} \mathrm{D}_{4}$ with constant depth concentration of deuterium. The conditions of the nuclear physics experiments and the methods of treating the generated spectra are reported in greater detail in [9].

\section{RESULTS AND DISCUSSION}

Under deuteron irradiation, the deuterium ion concentration, $C_{\mathrm{D}}$, increased, and linear dependences were observed between $C_{\mathrm{D}}$ and the irradiation dose $N_{\mathrm{d}}$. To find deuterium concentrations $C_{\text {Din }}$ in unirradiated samples, the measurement results were extrapolated to an irradiation dose equal to zero. The average concentration of deuterium ions $C_{\text {Din }}$ determined in this way

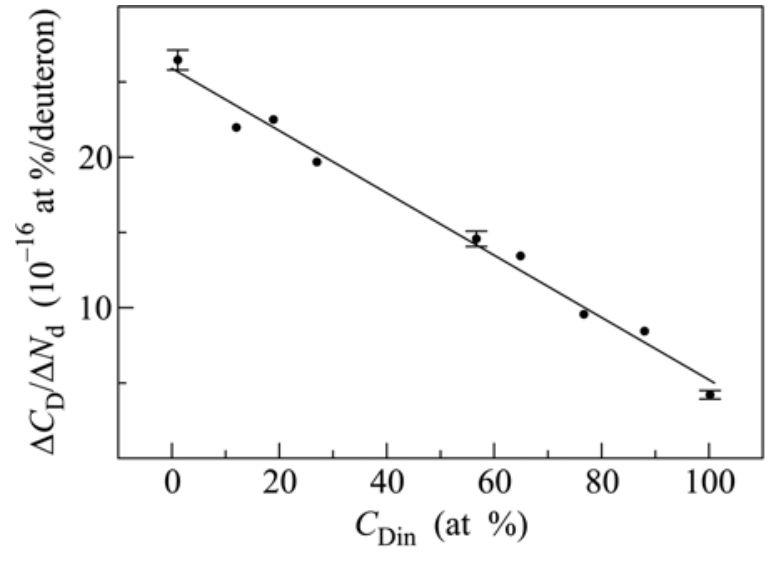

Fig. 2. Ratio $\Delta C_{\mathrm{D}} / \Delta N_{\mathrm{d}}$ in $\mathrm{BaZr}_{0.9} \mathrm{Y}_{0.1} \mathrm{O}_{3-\delta}$ micropowder versus the initial concentration of dissolved deuterium $C_{\text {Din }}$.

in the zone of analysis in a sample treated in heavy water vapor was $100.5 \pm 0.2$ at \%, i.e., as expected, the structural oxygen vacancies were completely filled with oxygen ions that arrived into the sample with water molecules. In the sample treated in water vapor with a natural mixture of hydrogen isotopes, the value of $C_{\text {Din }}$ was $1.1 \pm 1.0$ at $\%$, i.e., it did not reliably differ from 0 .

As it is shown in Fig. 2, a linear dependence of $\Delta C_{\mathrm{D}} / \Delta N_{\mathrm{d}}$ on $C_{\text {Din }}$ was observed. This result is satisfactorily described by equation (3) derived with an assumption of the hydrogen isotope exchange between accelerator beam deuterons and dissolved protium ions. Thus, the hypothesis of a new type of hydrogen isotope exchange was confirmed. Furthermore, it can be seen in Fig. 2 that, besides the isotope exchange, there is another mechanism causing deuterium accumulation in the oxide under deuteron irradiation. This is confirmed by the value of $\Delta C_{\mathrm{D}} / \Delta N_{\mathrm{d}}$ reliably distinct from zero when $C_{\text {Din }}=100$ at $\%$. Probably it can be the case of an ordinary implantation. This effect has no relation to the presence or absence of dissolved protium and deuterium ions in the oxide. We irradiated with deuterons the oxide micropowders $\mathrm{BaZr}_{0.9} \mathrm{Y}_{0.1} \mathrm{O}_{3-\delta}$ and $\mathrm{BaZrO}_{3}$, which contained no dissolved $\mathrm{D}^{+}$or $\mathrm{H}^{+}$ions. In these experiments the values of $\Delta C_{\mathrm{D}} / \Delta N_{\mathrm{d}}$ were almost as low as during deuteron irradiation of the powder $\mathrm{BaZr}_{0.9} \mathrm{Y}_{0.1} \mathrm{O}_{3-\delta}$ treated in heavy-water vapors.

In this study we also obtained information about the properties of the isotope exchange in samples exposed to proton irradiation. It was established that the replacement of $\mathrm{D}^{+}$by $\mathrm{H}^{+}$ions occurred with a very low intensity, approximately two orders of magnitude smaller than that during replacement of $\mathrm{H}+$ by $\mathrm{D}^{+}$ ions under deuteron irradiation. In these conditions, the experimental data during proton irradiation were found only for the powder with the maximum initial concentration of dissolved deuterium $C_{\mathrm{Din}}=100.5$ at $\%$. 
The value $\left|\Delta C_{\mathrm{D}} / \Delta N_{\mathrm{p}}\right|=(0.20 \pm 0.04) \times 10^{-16}$ at \%/proton was obtained. Here it is given with regard to the correction for the increased deuterium concentration during measurements of $C_{\mathrm{D}}$ with the use of the reaction ${ }^{2} \mathrm{H}(\mathrm{d}, \mathrm{p}){ }^{3} \mathrm{H}$. The correction was $23 \%$.

A further research is needed for detailed interpretation of these results on the hydrogen isotope exchange of the new type. We can perform some simple estimates now. First of all, we should point out that the hydrogen isotope exchange observed in this work is not related to nuclear interactions between protium and deuterium ions. This is certified by the level of $\Delta C_{\mathrm{D}} / \Delta N_{\mathrm{d}}$ value registered in the study. The estimates obtained with the use of the data presented in Fig. 2 show that the $0.2 \mathrm{H} / \mathrm{D}$ exchange event occurred in this volume when one deuteron enters into the sample. The probability of a nuclear interaction is usually several powers lower [10].

Further, it was of interest to analyze the results in the model assuming reversibility of the isotope exchange. In this approximation for the probabilities $k_{1}$ and $k_{2}$ in equations (3), (4) the following relationships were assumed:

$$
k_{1} \propto \exp \left(\Delta E / k_{\mathrm{B}} T\right) \text { and } k_{2} \propto \exp \left(-\Delta E / k_{\mathrm{B}} T\right),
$$

where $k_{\mathrm{B}}$ is the Boltzmann constant, $T$ is the absolute temperature, and $\Delta E=E_{\mathrm{H}}-E_{\mathrm{D}}$ is the difference between the equilibrium energies of protium $\left(E_{\mathrm{H}}\right)$ and deuterium $\left(E_{\mathrm{D}}\right)$ ions in the oxide. In this case, for the determination of the difference in the energies $\Delta E$ of protium and deuterium ions, we can use the equation

$$
\frac{\Delta C_{\mathrm{D}} / \Delta N_{\mathrm{d}}}{\left|\Delta C_{\mathrm{D}} / \Delta N_{\mathrm{p}}\right|}=\exp \left(\frac{2 \Delta E}{k_{\mathrm{B}} T}\right)
$$

where the values of $\Delta C_{\mathrm{D}} / \Delta N_{\mathrm{d}}$ and $\left|\Delta C_{\mathrm{D}} / \Delta N_{\mathrm{p}}\right|$ correspond to the irradiation of the samples containing only dissolved ions of protium or deuterium, respectively. Here we considered the contribution to $\Delta C_{\mathrm{D}} / \Delta N_{\mathrm{d}}$ only from $H / D$ isotope exchange. This contribution prevailed during deuteron irradiation and was $(22.5 \pm 2.5) \times$ $10^{-16}$ at $\%$ /deuteron. In our estimates using equation (6) we supposed that the temperature in the irradiation area was higher than room temperature, but we made no special measurements of the temperature. The temperature of the target was assumed to be $T=350 \mathrm{~K}$. We took into account that the sample subjected to irradiation can be heated by several tens of degrees $[9,11]$. The irradiation conditions in $[9,11]$ were close to ours. In future works, the effects of heating in the irradiation area should be determined. As a result, we found $\Delta E=0.071 \pm 0.003 \mathrm{eV}$.

The obtained $\Delta E$ result was compared with the data from study [1] on the direct measurements of the H/D thermodynamic effect, $C_{\mathrm{D}} / C_{\mathrm{H}}$ in the solubility of water vapor in $\mathrm{BaZr}_{0.9} \mathrm{Y}_{0.1} \mathrm{O}_{3-\delta}$ under equal partial pressures of $\mathrm{H}_{2} \mathrm{O}$ and $\mathrm{D}_{2} \mathrm{O}$ vapors. These measurements were carried out at $T=573 \mathrm{~K}$, and the value
$C_{\mathrm{D}} / C_{\mathrm{H}}=1.8 \pm 0.2$ was derived. On the other hand, the value of thermodynamic isotope effect $C_{\mathrm{D}} / C_{\mathrm{H}}$ on the solubility estimated with the value of $\Delta E=0.071 \mathrm{eV}$ obtained in our study under the same conditions was $1.64 \pm 0.05$. It is seen that the values of isotope effect in solubility determined by the direct method and the new type of the hydrogen isotope exchange are similar. It shows that the investigation of this new type of the hydrogen isotope exchange can be used for obtaining data on the equilibrium states of hydrogen ions in oxides. Note that our experiments were carried out at near-room temperature. At such low temperatures, the solubility of hydrogen isotopes in oxides cannot be measured directly.

\section{CONCLUSIONS}

Thus, our results show clearly that the hydrogen isotope exchange is realized in proton-conducting oxides during proton and deuteron irradiation. This type of exchange is promising for obtaining new information about the state, solubility and diffusion of hydrogen isotopes in oxides. Unlike the other types of isotope exchange, this new one was observed in the conditions when the rates of replacement of $\mathrm{H}^{+}$by $\mathrm{D}^{+}$ and of $\mathrm{D}^{+}$by $\mathrm{H}^{+}$were determined by the processes occurring inside the crystal, while any appreciable effect of conjugate diffusion of oxygen and hydrogen ions and of the interface processes were eliminated.

This work was supported by the Presidium of the Russian Academy of Sciences (project "Hydrogen Isotopes in Oxides").

\section{REFERENCES}

1. V. I. Tsidilkovskii, V. B. Vykhodets, T. E. Kurennykh, et al., JETP Lett. 92, 774 (2010).

2. K. Morita, B. Tsuchiya, S. Nagata, et al., Nucl. Instrum. Methods Phys. Res. B 249, 322 (2006).

3. B. Tsuchiya, E. Iizuka, K. Soda, et al., J. Nucl. Mater. 258-263, 555 (1998).

4. E. Iizuka, T. Horikawa, B. Tsuchiya, et al., Jpn. J. Appl. Phys., Pt 1 40, 3343 (2001).

5. K. D. Kreuer, Ann. Rev. Mater. Res. 33, 333 (2003).

6. The Stopping and Ranges of Ions in Matter, SRIM2013. http://www.srim.org

7. J. F. Ziegler, J. P. Biersack, and U. Littmark, The Stopping and Range of Ions in Solids (Pergamon, New York, 1984), vol. 1.

8. V. B. Vykhodets, E. V. Vykhodets, B. A. Gizhevskii, et al., JETP Lett. 87, 115 (2008).

9. V. B. Vykhodets, S. M. Klotsman, T. E. Kurennykh, et al., Fiz. Met. Metalloved. 63, 974 (1987).

10. Ion Beam Analysis Nuclear Data Library (IBANDL), International Atomic Energy Agency, Nuclear Data Section. http://www-nds.iaea.org

11. V. Kh. Alimov, B. Tyburska-Püschel, M. H. J. t Hoen, et al., Phys. Scripta T 145, 014037 (2011). 